\title{
Day-Ahead Probabilistic Load Flow Analysis Considering Wind Power Forecast Error Correlation
}

\author{
Qiang Ding1, Chuancheng Zhang1, Jingyang Zhou ${ }^{1}$, Sai Dai' ${ }^{1}$, Dan Xu1 ${ }^{1}$, Zhiqiang Luo², \\ Chengwei Zhai ${ }^{3}$ \\ ${ }^{1}$ Power Automation Department, China Electric Power Research Institute, Beijing, China \\ ${ }^{2}$ National Electric Power Dispatch and Control Center, State Grid Corporation of China, Beijing, China \\ ${ }^{3}$ Electrical and Electronic Engineering College, Zhejiang University, Hangzhou, China \\ Email: dingqiang@epri.sgcc.com.cn,luo-zhiqiang@sgcc.com.cn,3110100655@zju.edu.cn
}

How to cite this paper: Ding, Q., Zhang, C.C., Zhou, J.Y., Dai, S., Xu, D., Luo, Z.Q. and Zhai, C.W. (2017) Day-Ahead Probabilistic Load Flow Analysis Considering Wind Power Forecast Error Correlation. Energy and Power Engineering, 9, 292-299. https://doi.org/10.4236/epe.2017.94B034

Received: February 21, 2017

Accepted: March 30, 2017

Published: April 6, 2017

\begin{abstract}
Short-term power flow analysis has a significant influence on day-ahead generation schedule. This paper proposes a time series model and prediction error distribution model of wind power output. With the consideration of wind speed and wind power output forecast error's correlation, the probabilistic distributions of transmission line flows during tomorrow's 96 time intervals are obtained using cumulants combined Gram-Charlier expansion method. The probability density function and cumulative distribution function of transmission lines on each time interval could provide scheduling planners with more accurate and comprehensive information. Simulation in IEEE 39-bus system demonstrates effectiveness of the proposed model and algorithm.
\end{abstract}

\section{Keywords}

Wind Power, Time Series Model, Forecast Error Distribution, Forecast Error Correlation, Probabilistic Load Flow, Gram-Charlier Expansion

\section{Introduction}

Recently, with the increase of wind power portion in power generation in China, how to conduct effective power flow analysis of power system including wind farm is always the focus of researchers. Day-ahead wind power prediction and probabilistic power flow analysis is valuable to guarantee the reliability and economy of short-term dispatch plan.

However, nowadays, for short-term dispatch plan including wind power, only 
a predicting time sequence is provided to operators which is not accurate enough for a reliable generation schedule. Most literature these days is focusing on wind power generation prediction, wind power prediction error, probabilistic power flow analysis based on long-term wind power models. And most literature relating to Gram-Charlier expansion is using this method with the Weibull distribution to analyze long-term probabilistic load flow, however, Weibull distribution's frequency spectrum is dispersed which makes it inappropriate to analyze short-term flow analysis.

Towards these problems, this paper puts forward a method for analyzing the probabilistic load flow on each time interval of day-ahead generation schedule based on prediction error distribution and Gram-Charlier expansion method. Combined with day-ahead wind speed prediction model and wind power prediction error's distribution which considering its correlation, this method provides more data and information support for a more advanced economic dispatch.

\section{Methodology}

\subsection{Overall Model}

This method starts from establishing a single wind farm's wind speed forecasting time series by day-ahead wind speed anticipation methods such as density forecast and regression analysis etc. In this thesis, ARMA combined time shift technique will be used to generate a simulated forecasting time series applying in example. More sophisticated wind speed anticipation method should be used in practical application. This step provides an expected value for following cumulants calculation and series expansion.

In fact, errors exist in wind speed and wind power output anticipation methods these days always. Using statistics methods, we can establish forecasting errors' probabilistic distribution models. This thesis adopts Beta distribution to fit forecasting errors' frequency distribution histogram. By establishing the model of wind power predicting errors' correlation complete the whole simulated scene. This thesis quantitative analyze the correlation of forecasting errors and qualitative analyze the correlation between forecasting wind power and wind power predicting errors. Meanwhile, by using covariance matrix transformation method to decouple correlated distributions solves the problem of only independent random variables can be used in DC probabilistic load flow calculation by series expansion method.

At last, obtaining each wind power predicting fluctuation's independent probabilistic distribution, by using Gram-Charlier expansion combined cumulants to calculate each power line's probabilistic load flow's distribution. Applying this method is both convenient and efficient in analyzing day-ahead probabilistic flow distribution. Figure 1 is the illustrative diagram of this article.

\subsection{ARMA Model \& Time Shifting Technic}

This thesis uses auto-regressive and moving average (ARMA) model combining 


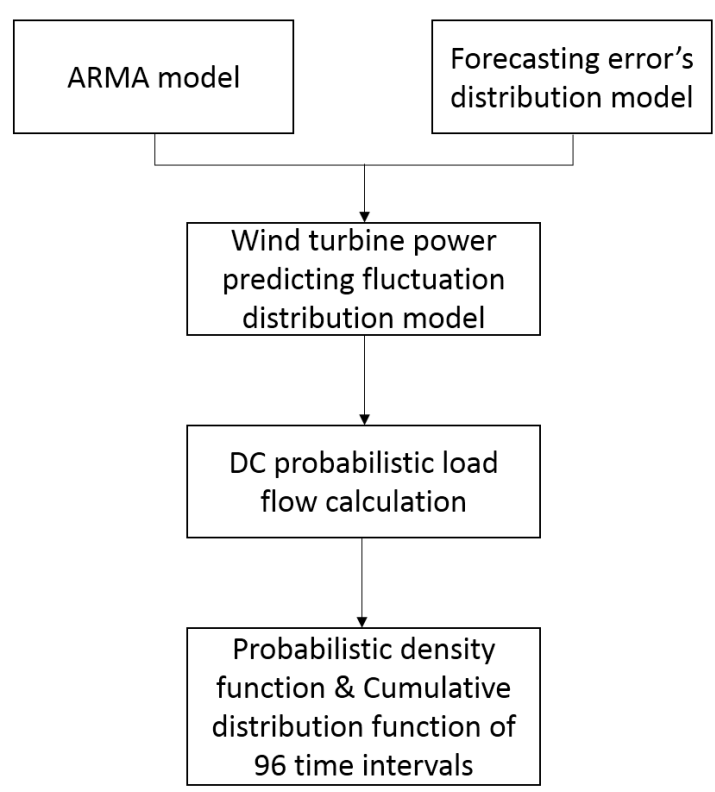

Figure 1. Illustrative diagram of day-ahead power flow analysis considering wind power output forecast error distribution.

time shifting technique to generate a time series considering wind speed's spatial correlation. Though ARMA model and time shifting technique can effectively predict wind speed and consider multiple wind farm's correlation, then use the wind turbine output model to acquire multiple wind power predicting model.

ARMA model is widely applied in establishing wind speed and wind speed prediction time series. The classic ARMA model can be described as the following equation:

$$
S_{t}=\sum_{i=1}^{n} \varphi_{i} S_{t-i}+\alpha_{t}-\sum_{j=1}^{n} \theta_{j} \alpha_{t-j}
$$

where $s_{t}$ is the time series value at time $t, \varphi_{i}$ and $\theta_{j}$ are the auto-regressive and moving average parameters of the model, respectively. $\alpha_{t}$ is a normal white noise process with zero mean and a variance of $\sigma_{2}$ as a normal independent distribution.

The hourly simulated wind speed $S W_{t}$ at time $t$ is obtained from the mean speed, its standard deviation and the time series $s_{t}$

$$
S W_{t}=\mu_{t}+\sigma_{t} S_{t}
$$

Thus, a single wind farm's day-ahead prediction wind speed time series is generated.

Then, by using the time shifting technique we can produce multiple wind farms' wind speed prediction time series with certain correlation value. Assume that the new wind speed time series designated as $s_{t}^{\prime}$ is to be produced from the original simulated series $s_{t}$ with a certain correlation coefficient $R$. Let $t=K+t_{0}, K$ is the integral part and $t_{0}$ is the fractional part of time shifting value $t$. The wind speed $s_{t}^{\prime}$ can be given by: 


$$
s^{\prime}{ }_{K+i}=\left(1-t_{0}\right) \times s_{i}+t_{0} \times s_{i+1}
$$

If each item's subscript value exceeds $n$, then replaces the value by its original value minus a $n$.

After obtaining multiple wind farm's wind speed time series, through the wind turbine output model we can get multiple wind power prediction time series model.

\subsection{Wind Power Prediction Error's Distribution Model}

This thesis uses non-standard Beta distribution to fit wind turbine output forecasting errors' distribution. This kind of probabilistic distribution shows a good reliability in wind turbine output intervals forecasting errors fit.

Non-standard Beta distribution's probabilistic density equation can be described as

$$
f(x, \gamma, \eta, a, b)=\left\{\begin{array}{cr}
\frac{1}{(b-a) \beta(\gamma, \eta)}\left(\frac{x-a}{b-a}\right)^{\gamma-1}\left(\frac{b-x}{b-a}\right)^{\eta-1} & a \leq x \leq b \\
0 & \text { other }
\end{array}\right.
$$

where $a$ and $b$ is the bound of value $x, \gamma$ and $\eta$ is shape parameter, defined as

$$
\left\{\begin{array}{l}
\gamma=\frac{\left(\mu_{x}-a\right)^{2}\left(b-\mu_{x}\right)-\sigma_{x}^{2}\left(\mu_{x}-a\right)}{\sigma_{x}^{2}(b-a)} \\
\eta=\frac{\left(\mu_{x}-a\right)\left(b-\mu_{x}\right)^{2}-\sigma_{x}^{2}\left(b-\mu_{x}\right)}{\sigma_{x}^{2}(b-a)}
\end{array}\right.
$$

where $\mu_{x}$ and $\sigma_{x}$ is the mean and standard deviation, respectively. Then, divide wind turbine output into several intervals

\subsection{Forecasting Errors Correlation Analysis}

There exists a correlation among multiple wind turbine output forecasting errors. With analysis of actual wind farm's historical wind turbine output's forecasting errors' time series we can calculate the correlation coefficient. There are several characteristics of forecasting errors correlation.

1) The cross-correlation value of multiple wind can be calculated with two wind farms' wind speed time series.

2) The correlation coefficient is influenced by the distance of wind farms, wind speed and wind direction. The nearer of wind farms, the higher of wind speed and the more similar of wind direction, the higher the forecasting error's correlation coefficient among wind farms.

3) The lower of wind speed, the higher of the auto-correlation coefficient but the lower of the cross correlation coefficient.

4) There exists a positive correlation between wind turbine output's predicting time series and its forecasting error.

Based on these characteristics, the model of wind turbine output forecasting error is established as following: 
1) When the predicting wind speed less than $10 \mathrm{~m} / \mathrm{s}$, consider the forecasting error distributions in this range are irrelevant.

2) When the predicting wind speed more than $10 \mathrm{~m} / \mathrm{s}$, consider the forecasting error distributions in this range as a moderate correlation.

3) Since there is little specific data about forecasting error's correlation between different wind turbine output intervals, consider distribution in different output intervals as irrelevant.

Then, we use covariance matrix transformation method to decouple distribution with correlation. The essence is to use the original forecasting error's covariance matrix's eigenvalue replaces as the new distribution's deviation, also as the second order cumulant.

\subsection{PLF Equation and Gram-Charlier Expansion}

The equation of DC probabilistic load flow (PLF) is

$$
P_{\text {line }}=T Z P=H P
$$

where $\boldsymbol{H}$ is the flow sensitivity matrix. Each element represents as when add 1 MW at bus $j$ then line $i k$ 's value. Matrix $\boldsymbol{H}$ is defined as

$$
H_{(i k) j}=\frac{Z_{i j}-Z_{k j}}{X_{i k}}
$$

If bus $j$ is the slack bus, then $H_{(i k) j}=0$.

If a probabilistic distribution's cumulants has been acquired, its distribution equation can be calculated with Gram-Charlier expansion as

$$
\begin{aligned}
& F(x)=\sum_{i=0}^{\infty} \frac{c_{i}}{i !} \Phi^{(i)}\left(\frac{x-\mu}{\sigma}\right) \\
& f(x)=\frac{1}{\sigma} \sum_{i=0}^{\infty} \frac{c_{i}}{i !} \varphi^{(i)}\left(\frac{x-\mu}{\sigma}\right)
\end{aligned}
$$

where $c_{i}$ is parameters.

Use the additivity and DC PLF equation, we can calculate the cumulants of each line as

$$
\gamma_{v}=h_{i 1}^{v} \gamma_{v}^{(1)}+h_{i 2}^{v} \gamma_{v}^{(2)}+\cdots+h_{i n}^{v} \gamma_{v}^{(n)}
$$

Then, each line's probabilistic density function and cumulative distribution function can be calculated by Gram-Charlier expansion.

\section{Case Study}

This thesis uses an improved IEEE 39-bus system to simulate as a case. This system has 39 buses and 46 power lines. Based on the original model, assume that bus $32,33,34$ are wind turbine output buses with a cut-in wind speed of $4 \mathrm{~m} / \mathrm{s}$, a rated wind speed of $12 \mathrm{~m} / \mathrm{s}$ and a cut-out wind speed of $20 \mathrm{~m} / \mathrm{s}$. With the model from this thesis, we can calculate out each power line flow's probabilistic density function and cumulative distribution function of tomorrow's 96 time intervals. In this case, set the forecasting error's correlation coefficient as 


$$
R=\left[\begin{array}{lll}
1.0 & 0.5 & 0.6 \\
0.5 & 1.0 & 0.4 \\
0.6 & 0.5 & 1.0
\end{array}\right]
$$

and use covariance matrix transformation method to correct the original cumulants values of these three wind turbine output buses.

Figure 2 and Figure 3 is line 5 - 6 flow's probabilistic density function and cumulative distribution function curves. And if the flow limit of line 5 - 6 is 1.2 per unit value, Figure 4 is its load flow limit violation probability graph.

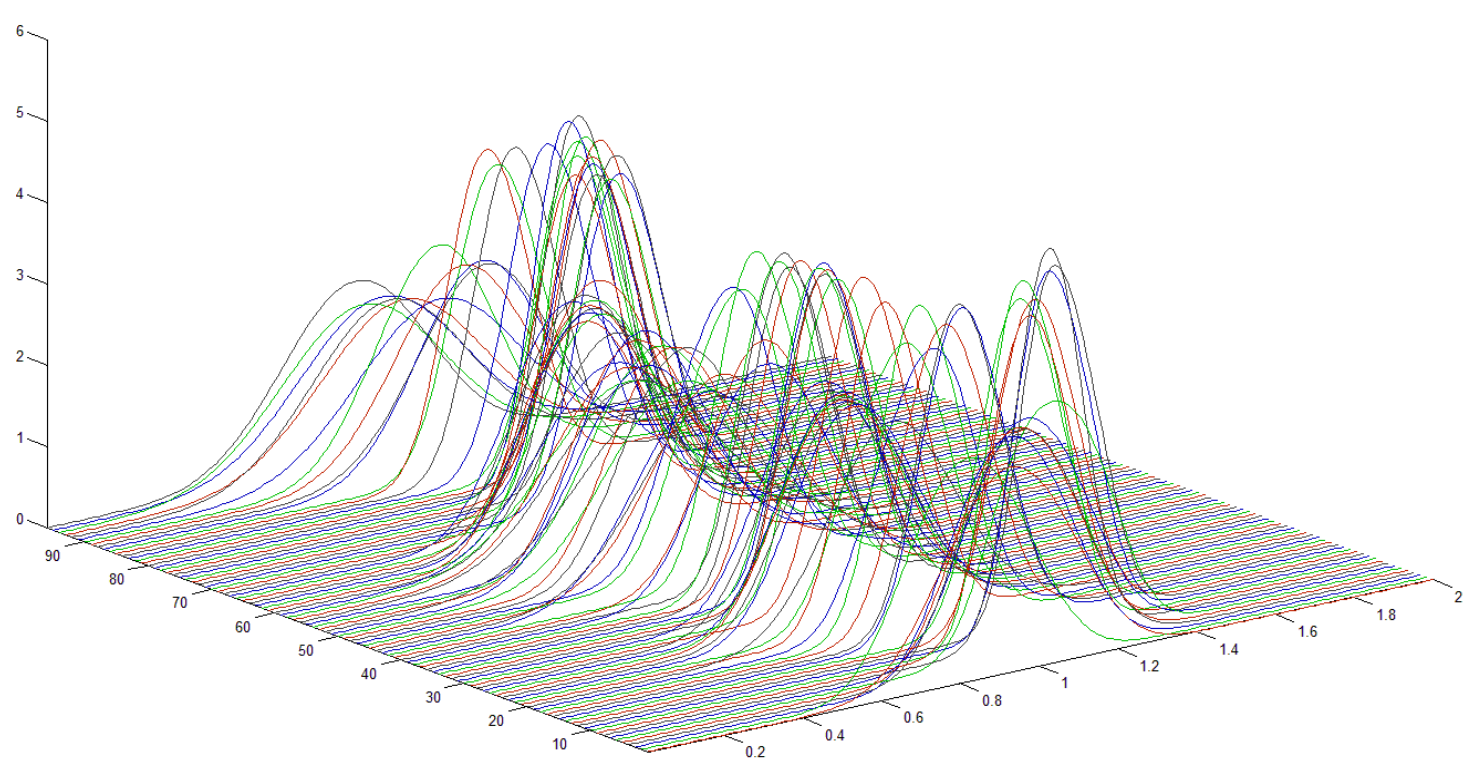

Figure 2. Probabilistic density curves of load flow on line 5 - 6 for tomorrow's 96 time intervals.

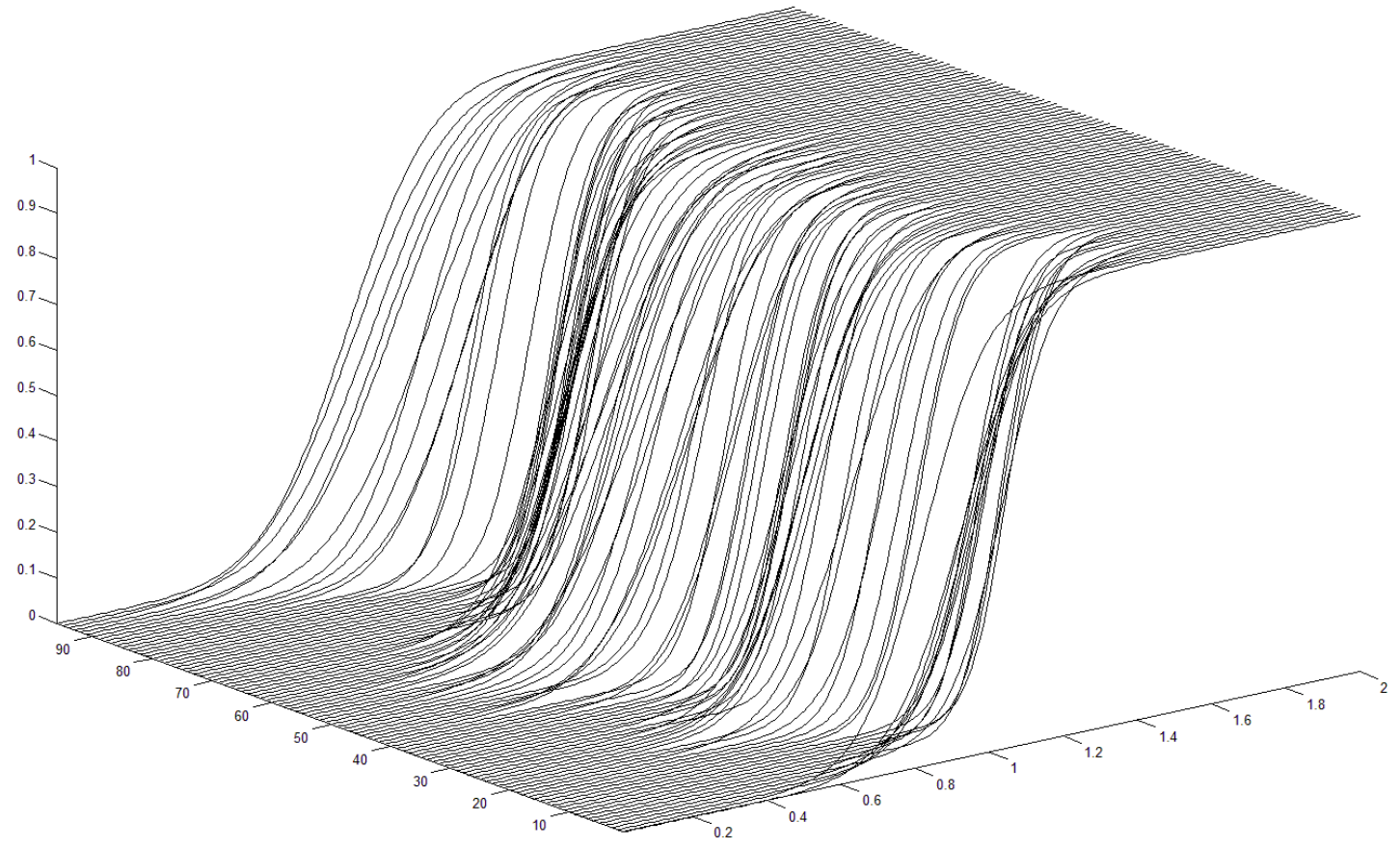

Figure 3. Cumulative distribution curves of load flow on line 5 - 6 for tomorrow's 96 time intervals. 


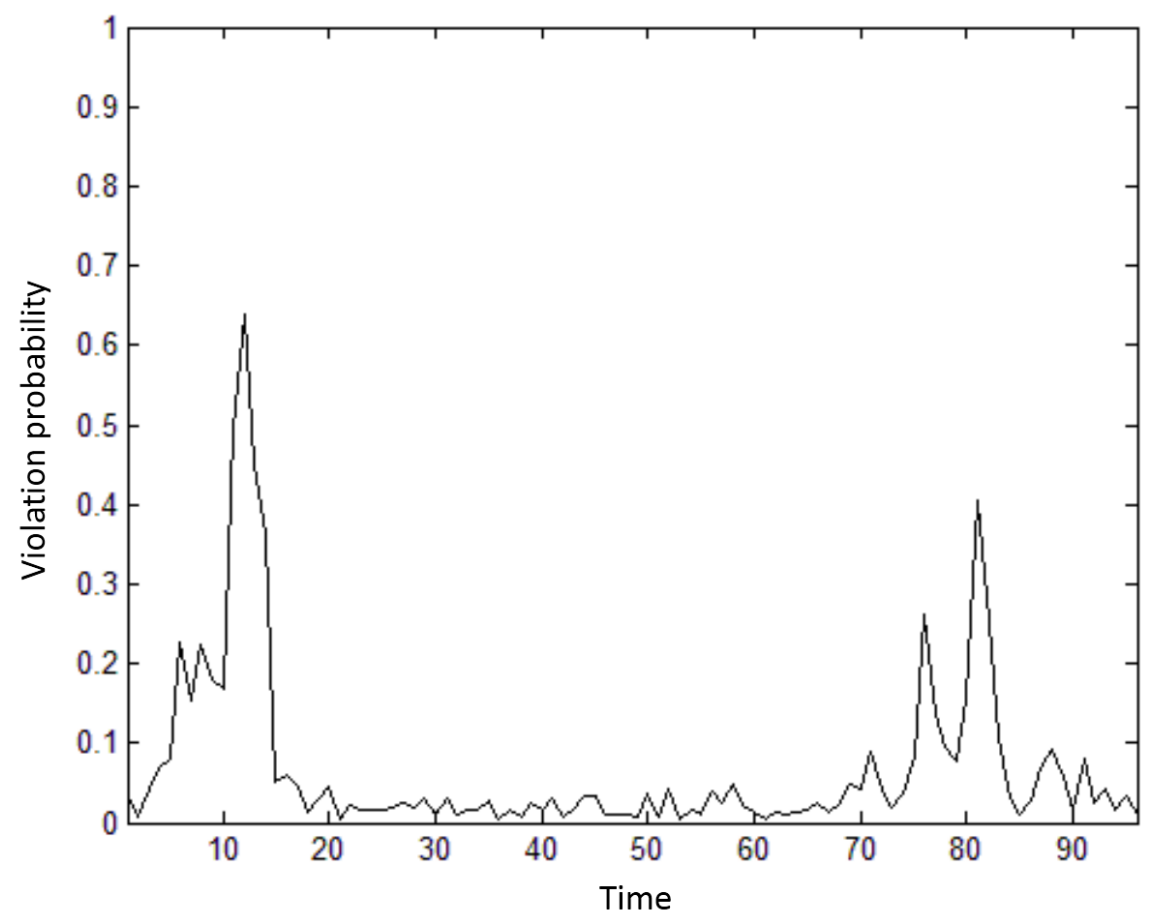

Figure 4. Load flow limit violation probability on line 5 - 6 for tomorrow's 96 time intervals.

\section{Conclusion}

This article puts forward a method based on prediction error's distribution and Gram-Charlier expansion to analyze every time intervals of tomorrow combining day-ahead wind speed prediction model and wind power prediction error's distribution considering its correlation. Based on every time interval's probabilistic density function and cumulative distribution function, load flow information with time series feature can be effectively acquired.

\section{Acknowledgements}

This work was financially supported by the Science and Technology Project of China State Grid Corp.

\section{References}

[1] Ding, H.J., Song, Y.H., Hu, Z.C., Wu, J.C. and Fan, X.X. (2013) Probability Density Function of Day-Ahead Wind Power Forecast Errors Based on Power Curves of Wind Farms. Proceedings of the CSEE, 33, 136-144.

[2] Liu, X.J. and Xie,C.Y. (2014)Wind Power Fluctuation Interval Estimation Based on Beta Distribution. Electric Power Automation Equipment, 34, 26-30.

[3] Lin, W.X., Wen, J.Y., Ai, X.M., Cheng, S.J. and Lee, W.J. (2012) Probability Density Function of Wind Power Variations. Proceedings of the CSEE, 32, 38-46.

[4] Xie, K.G. and Billinton, R. (2009) Considering Wind Speed Correlation of WECS in Reliability Evaluation Using the Time-Shifting Technique. Electric Power Systems Research, 79, 687-693.https://doi.org/10.1016/j.epsr.2008.10.013

[5] Girard, R. and Allard, D. (2012) Spatio-temporal Propagation of Wind Power Pre- 
diction Errors. Wind Energy, 16,999-1012.https://doi.org/10.1002/we.1527

[6] Zhang, P. and Lee, S. (2004) Probabilistic Load Flow Computation Using the Method of Combined Cumulants and Gram-Charlier Expansion.IEEE Transactions on Power Systems, 19, 676-682.https://doi.org/10.1109/TPWRS.2003.818743

[7] Zhang, K.F., Yang, G.Q., Chen, H.Y. and Ding, Q. (2014) AnEstimate Method for Wind Power Forecast Errors Based on Numerical Feature Extraction. Automation of Electric Power Systems,38, 22-28.

[8] Ye, L. and Zhao, Y.N. (2014) A review on Wind Power Prediction Based on Spatial Correlation Approach. Automation of Electric Power Systems, 38, 126-134.

[9] Zhang, J.Z. and Cheng, M. (2012) Modeling of Time Series Stochastic Wind Speed for Wind Power Generation. Technology, 1,58-61.

[10] Tang, B., Chen, B., Qu, Z.H., et al. (2014) Research of Day-Ahead Forecasting Model for Wind Power Based on Time Series and Its Application. Water Resources and Power, 32,193-196.

Submit or recommend next manuscript to SCIRP and we will provide best service for you:

Accepting pre-submission inquiries through Email, Facebook, LinkedIn, Twitter, etc. A wide selection of journals (inclusive of 9 subjects, more than 200 journals) Providing 24-hour high-quality service User-friendly online submission system Fair and swift peer-review system Efficient typesetting and proofreading procedure Display of the result of downloads and visits, as well as the number of cited articles Maximum dissemination of your research work

Submit your manuscript at: http://papersubmission.scirp.org/

Or contact epe@scirp.org 